\title{
Spin relaxation of atoms in molecular buffer gases
}

\section{Citation}

Berg, Howard C. 1965. Spin relaxation of atoms in molecular buffer gases. Journal of Chemical Physics 43, no. 5: 1851.

\section{Published Version}

http://dx.doi.org/10.1063/1.1697036

\section{Permanent link}

http://nrs.harvard.edu/urn-3:HUL.InstRepos:4696186

\section{Terms of Use}

This article was downloaded from Harvard University's DASH repository, and is made available under the terms and conditions applicable to Other Posted Material, as set forth at http:// nrs.harvard.edu/urn-3:HUL.InstRepos:dash.current.terms-of-use\#LAA

\section{Share Your Story}

The Harvard community has made this article openly available.

Please share how this access benefits you. Submit a story.

Accessibility 
possibility of an involvement of excited species other than free atoms should not be overlooked although it is unlikely that definitive conclusions will be possible before considerable further experimental information becomes available.

${ }^{1}$ H. Yamazaki and R. J. Cvetanović, J. Chem. Phys. 39, 1902 (1963).

${ }^{2}$ H. Yamazaki and R. J. Cvetanović, J. Chem. Phys. 40, 582 (1964).

${ }^{3} \mathrm{H}$. Yamazaki and R. J. Cvetanović, J. Chem. Phys. 41, 3703 (1964).

${ }_{4}^{4}$ P. Warneck, Discussions Faraday Soc. 37, 57 (1964).

5 P. Warneck, J. Chem. Phys. 43, 1849 (1965).

${ }^{6}$ K. F. Preston and R. J. Cvetanovic (to be published).

\section{Spin Relaxation of Atoms in Molecular Buffer Gases}

\author{
HowARD C. BERG* \\ Lyman Laboratory of Physics, Harvard University \\ Cambridge, Massachusetts
}

(Received 9 March 1965)

I $\mathrm{N}$ his discussion of the magnetic relaxation of optically pumped rubidium in a buffer gas containing molecules with large electric-dipole moments, $\mathrm{NcNeal}^{1}$ develops a theory in which a static electric field couples the two states ${ }^{2} S_{\frac{1}{3}}\left(M_{J}= \pm \frac{1}{2}\right)$ in the limit of zero magnetic field. This is forbidden by Kramers' theorem (time-reversal invariance) ${ }^{2,3}$ The error arises from the use of stationary perturbation theory in the computation of a nonadiabatic effect.

Let an atom in the state $m={ }^{2} S_{\frac{1}{2}}\left(M_{J}=\frac{1}{2}\right)$, which is at rest in a magnetic field $H$ oriented along the $z$ axis, be exposed to a uniform electric field $\mathbf{E}$ (not parallel to the coordinate axes) for a period of time $\tau \ll\left(g_{J} \mu_{0} H / \hbar\right)^{-1}$, where $g_{J}$ is the electron $g$ factor and $\mu_{0}$ is the Bohr magneton. If we treat the electric field classically and ignore the magnetic field arising from the time rate of change of the electric field, then the probability that a transition occurs between the state $m$ and the state $k={ }^{2} S_{\frac{1}{2}}\left(M_{J}=-\frac{1}{2}\right)$ is given in second-order timedependent perturbation theory by

$$
P_{m k} \sim \tau_{\hbar^{2}}\left|\sum_{i \neq m} \frac{\left\langle k\left|\mathcal{K}^{\prime}\right| i\right\rangle\left\langle i\left|\mathcal{F}^{\prime}\right| m\right\rangle}{\left(E_{m}-E_{i}\right)}\right|^{2},
$$

where $\mathfrak{H}^{\prime}$ is the electric-dipole interaction defined by McNeal, and the summation is over the excited $p$ states of the atom, which belong to the energy eigenvalues $E_{i}$. The sum which appears in this expression is $\left(E_{m}-E_{k}\right) C_{k}^{(2)}=g_{J} \mu_{0} H C_{k}^{(2)}$, where $C_{k}^{(2)}$ is the stationary-perturbation-theory coefficient evaluated by McNeal. Since $C_{k}{ }^{(2)}$ is independent of $H, P_{m k} \rightarrow 0$ as $H \rightarrow 0$, as required by Kramers' theorem.

McNeal discusses the transition probability in terms of an "effective magnetic field," that is, the magnetic field which, when applied in the $x$ direction for a time $\tau$, yields the same transition probability $P_{m k}$. This field is $2\left|C_{k}{ }^{(2)}\right| H$, which is smaller than $H$. For a collision between rubidium and a molecule of dipole moment $1.5 \mathrm{D}$ at $H=0.6 \mathrm{G}, P_{m k}$ is about $10^{-12}$, so the electricfield mechanism does not explain the experimental results.

The author wishes to thank J. H. Van Vleck for discussions of this problem.

* Junior Fellow, Harvard Society of Fellows.

1 R. J. McNeal, J. Chem. Phys. 40, 1089 (1964).

${ }^{2}$ H. A. Kramers, Proc. Roy. Acad. Amsterdam 33, 959 (1930); see L. D. Landau and E. M. Lifshitz, Quantum Mechanics (Addison-Wesley Publishing Company, Inc., Reading, Massachusetts, 1958), p. 202.

${ }^{3}$ Spin transitions due to oscillating electric fields are discussed in the theory of spin-lattice relaxation of paramagnetic salts. See R. de L. Kronig, Physica 6, 33 (1939); J. H. Van Vleck, Phys. Rev. 57, 426 (1940); and R. Orbach, Proc. Phys. Soc. (London) 77, 821 (1961); Proc. Roy. Soc. (London) A264, 458, 485 (1961).

\section{Spin Relaxation of Rubidium in Molecular Buffer Gases}

\author{
R. J. MCNeaL \\ Aerospace Corporation, El Segundo, California
}

(Received 9 April 1965)

$\mathrm{N}$ the preceeding comment ${ }^{1}$ Berg asserts that a previous calculation ${ }^{2}$ of the spin-relaxation cross section of optically oriented rubidium atoms in a buffer gas containing molecules with large electric-dipole moments is in error because of the use of stationary perturbation theory in the calculation of a nonadiabatic effect. Time-dependent perturbation theory was used, however, in the previous calculation, although in a less direct way than in Berg's Eq. (1). The error in the previous calculation is in Eq. (24) of Ref. 2, which should be written

$$
\left|\mathbf{H}_{1}\right|^{2} \alpha|\mathbf{H}|^{2}\left|C_{k}{ }^{(2)}\right|^{2},
$$

where $\left|\mathbf{H}_{1}\right|$ is the magnitude of the effective magnetic field and $|\mathbf{H}|$ is the magnitude of the applied Zeeman field in the experiment. The calculation in Ref. 2 is then essentially identical to Berg's. Both calculations yield zero cross section in zero magnetic field in agreement with Kramer's theorem. The author is indebted to Berg for pointing out the discrepancy between his calculation and the author's.

A correct treatment of the polar molecule-oriented rubidium collision is not possible, therefore, when the collision is simply represented by a time-dependent electric field. The collision complex must be treated on a molecular basis, and the effective Hamiltonian leading to a spin reorientation must be derived explicitly. Among the magnetic interactions arising during the collisions, Bernheim ${ }^{3}$ has proposed an intermolecular spin-rotation interaction to account for the relaxation in rare-gas collisions. Herman ${ }^{4}$ has done detailed calculations for this interaction, and the results agree well with experimental rare-gas cross sections. His calculation can be extended to polar buffer gases by including the induction term in the intermolecular potential, but 
the resulting cross section is too small to account for the observed result.

Large disorientation cross sections (in the range $10^{-17}-10^{-20} \mathrm{~cm}^{2}$ ) have been observed for ammonia, dimethyl ether, and benzene. In a gas containing the latter molecule, a large, negative pressure shift of the $\left(F=2, M_{F}=0\right) \rightarrow\left(F=1, M_{F}=0\right)$ hyperfine transition frequency of the ${ }^{87} \mathrm{Rb}$ ground state has been observed and attributed to a partial and transient electron transfer from rubidium to the benzene molecule during the collision. ${ }^{5}$ If such an electron transfer occurs also for the two polar molecules, then the large disorientation cross sections might result from interactions of the electron during association with the buffer-gas molecule rather than from intermolecular spin-rotation coupling.

${ }^{1}$ H. C. Berg, J. Chem. Phys. 43, 1851 (1965).

2 R. J. McNeal, J. Chem. Phys. 40, 1089 (1964).

${ }^{8}$ R. A. Bernheim, J. Chem. Phys. 36, 135 (1962).

${ }^{4}$ R. M. Herman, Phys. Rev. 136, A1576 (1964).

5 R. G. Brewer, J. Chem. Phys. 40, 1077 (1964).

On the Temperature Dependence of Cooperative Relaxation Properties in GlassForming Liquids-Comment on a Paper by Adam and Gibbs

\author{
MarTin Goldstein \\ Ceramics Division, Department of Metallurgy and Fibers and \\ Polymers Division, Department of Mechanical Engineering, \\ Massachusetts Institute of Technology \\ Cambridge, Massachusetts
}

(Received 20 May 1965)

$\mathbf{A}^{\mathrm{N}}$ $\mathrm{N}$ equation has been derived by Adam and Gibbs ${ }^{1}$ for the temperature dependence of the transition probability determining relaxation or flow of the form $W(T)=A \exp \left(-C / T S_{c}\right), A$ being a temperature-insensitive frequency factor, $C$ dimensionally an energy, and $S_{c}$ the excess configurational entropy of the liquid, a quantity whose changes with $T$ or $P$ are experimentally measurable ${ }^{2}$ and whose magn tude is asserted, in a thermodynamic theory of the glass transition, ${ }^{3}$ to vanish at a temperature $T_{2}$, lying somewhat below the experimental $T_{g}$. They have further shown that this relation leads to an equation similar in form to the WLF equation ${ }^{4}$ and experimentally indistinguishable from it, and that the calorimetrically determined $S_{c}$ agrees with the value derived from the temperature dependence of relaxation processes. The ratio $T_{a} / T_{2}$ appears to have a nearly universal value of 1.30 .

It is the purpose of this comment to point out three further types of experiments for which the Adam-Gibbs equation gives either quantitative or qualitative agreement, which the free-volume theory notably fails to do: these are pressure dependence, volume relaxation below $T_{\theta}$, and plasticity.

O'Reilly ${ }^{5}$ has shown that the pressure coefficient of $T_{q}$ is approximately equal to $T V \Delta \alpha / \Delta C_{\mathrm{P}}$, but only about half as great as $\Delta \beta / \Delta \alpha$ (see Ref. 2 or 5 for definitions). Additional evidence that this is an almost general situation was reviewed by the author. ${ }^{2}$ In the author's earlier paper ${ }^{2}$ it was shown that the experimental situation $d T_{a} / d P=T V \Delta \alpha / \Delta C_{\mathrm{P}}<\Delta \beta / \Delta \alpha$ implies that free volume cannot determine relaxation times, but either configurational entropy or enthalpy could. It seemed then that the approximate universality of $\Delta C_{\mathrm{P}}$ as contrasted with the variability of $\Delta C_{\mathrm{P}} / T_{g}$ implied the WLF equation could not be deduced from an entropy criterion, but could from an enthalpy one. It appears however that, if relaxation times are a function of the product $T S_{c}$, the WLF equation can be derived; it is further true that a nearly correct pressure dependence can be deduced: on the assumption of a negligible activation volume (see, however, below) and a constant $\Delta C_{\mathrm{P}}$ down to $T_{2}$ the following results:

$$
d T_{g} / d P=T_{2} V \Delta \alpha / \Delta C_{\mathrm{P}} .
$$

The difference between this and O'Reilly's equation applied near $T_{g}$ is about $30 \%$; it is not clear that the experimental data are precise enough to make the distinction, or for that matter whether quantitative agreement of a greater degree than this should indeed be demanded of the theory.

The Adam-Gibbs formula contains both an explicit dependence on temperature and an implicit one through $S_{c}$, the latter reflecting the changing structure of the liquid. It has been pointed out that experimental studies of volume flow in the glass transition region, where the structure can be temporarily frozen in while temperature is changed, permit a separation of a direct from an implicit effect of temperature ${ }^{6}$; a role for both is found; the relative importance of each depends on the particular substance; structure dominates in polyvinyl acetate, ${ }^{7}$ but the two being of about equal importance in borosilicate glass ${ }^{6}$ and zinc chloride. ${ }^{8}$ The ratio of the structural to the direct effect can be shown from the AdamGibbs equation to be $\Delta C_{\mathrm{P}} / S_{c}$, whose universal value is about 3. This agrees well with the measurements on polyvinyl acetate both in shear ${ }^{9}$ and volume. ${ }^{7}$ There are no experimental data on $\Delta C_{P}$ or $S_{c}$ in either borosilicate glass or zinc chloride. In any event the qualitative correctness of the Adam-Gibbs equation is to be noted, in contrast to the need in free-volume theories to introduce quite ad hoc a temperature dependence of free volume below $T_{g}$.

The Adam-Gibbs equation can be viewed as a reversion to the idea of viscosity as a thermally activated process, ${ }^{10}$ but with the rapidly changing activation energy near $T_{0}$ explained by the changing structure. Their derivation proceeds through the calculation of the free energy of an activated state, which therefore contains a volume of activation $V^{*}$ as well as an energy of activation: $W(T)$ is thus more generally written as

$$
\frac{A}{\exp \left[-(C+D P) / T S_{c}\right]},
$$

